\title{
Acute Pancreatitis after Biliary and Pancreatic Stent Removal with a Forward-Viewing Endoscope
}

\author{
Takeshi Okamoto ${ }^{a, b}$ Katsuyuki Fukudab \\ aDepartment of Hepato-Biliary-Pancreatic Medicine, Cancer Institute Hospital of Japanese \\ Foundation for Cancer Research, Tokyo, Japan; 'bepartment of Gastroenterology, St. Luke's \\ International Hospital, Tokyo, Japan
}

\section{Keywords}

Acute pancreatitis - Endoscopic retrograde cholangiopancreatography $\cdot$ Cholangitis · Case report

\begin{abstract}
Plastic biliary stents are commonly used for biliary drainage, while plastic pancreatic stents may be used prophylactically against acute pancreatitis in patients at high risk for post-endoscopic retrograde cholangiopancreatography pancreatitis. Removal of these prostheses is generally safe and can easily be performed in the ambulatory setting. Herein, we report a case of acute pancreatitis induced by removal of plastic biliary and pancreatic stents with a forward-viewing endoscope.
\end{abstract}

\section{Introduction}

Endoscopic retrograde cholangiopancreatography (ERCP) has become a standard procedure for the treatment of common bile duct stones and other causes of bile duct obstruction. While biliary drainage can also be achieved percutaneously, ERCP is generally preferred due to its high success rates, shorter hospital stays, and better quality of life based on its ability to avoid the use of percutaneous drainage tubes. On the other hand, difficulties in bile duct cannulation may lead to repeated contact with the ampulla causing temporary edema, as well as to unintended guidewire insertion or contrast injection into the pancreatic duct, setting the stage for post-ERCP pancreatitis (PEP).

PEP is one of the most feared complications of ERCP. The cause of PEP is believed to be multifactorial, with various known risk factors including pancreatography, long procedural 
time, pancreatic tissue sampling, intraductal ultrasonography, and difficult cannulation [1]. Pancreatic stent placement has been shown to reduce PEP [1, 2].

Biliary or pancreatic plastic stents placed during ERCP must subsequently be removed or replaced due to the risk of stent occlusion, with the exception of pancreatic stents without internal flanges which are designed to migrate spontaneously. Stent replacement is conducted using a duodenoscope, while simple removal may be performed in the ambulatory setting with a forward-looking endoscope. We report a case of acute pancreatitis occurring $3 \mathrm{~h}$ after biliary and pancreatic stent removal with a forward-looking endoscope.

\section{Case Report/Case Presentation}

An 83-year-old bedridden woman with a history of cerebral infarction, diabetes mellitus, hypertension, and urinary tract infections presented after 3 days of low-grade fever and dark urine. Medications included aspirin, levetiracetam, furosemide, and insulin glargine. She had no history of alcohol use or smoking.

Upon presentation, the patient was in mild distress. Vital signs were stable, with a temperature of $36.6^{\circ} \mathrm{C}$, blood pressure of $144 / 70 \mathrm{~mm} \mathrm{Hg}$, heart rate of $84 \mathrm{bpm}$, respiratory rate of 18 breaths per minute, and saturation of $93 \%$ on room air. Physical examination was unremarkable, with no abdominal tenderness or Murphy's sign. Laboratory results revealed elevated hepatobiliary enzymes (total bilirubin of $2.1 \mathrm{mg} / \mathrm{dL}$, aspartate aminotransferase of $367 \mathrm{U} / \mathrm{L}$, alanine aminotransferase of $525 \mathrm{U} / \mathrm{L}$, alkaline phosphatase of $443 \mathrm{U} / \mathrm{L}$, and gammaglutamyl transferase of $339 \mathrm{U} / \mathrm{L})$ with a slight increase in C-reactive protein $(21.9 \mathrm{mg} / \mathrm{L})$. No clear signs of bile duct obstruction could be identified on ultrasonography and CT with contrast.

The patient was admitted with the clinical diagnosis of acute cholangitis. ERCP was performed on the same day. After multiple unintended guidewire advancement into the pancreatic duct, endoscopic pancreatic sphincterotomy was performed using CleverCut 3V (Olympus Medical Systems, Tokyo, Japan). Successful bile duct cannulation was achieved. A small common bile duct stone was removed with a stone extraction balloon. Epinephrine was injected to achieve hemostasis following minor bleeding after stone extraction. A 7-Fr, 10-cm pigtail-type plastic biliary stent and a 5-Fr, 5-cm straight plastic pancreatic stent were placed for prophylaxis against bile duct stone recurrence and PEP (Fig. 1). The post-procedural course was uneventful, and the patient was discharged 4 days later.

After missing several appointments, the patient returned 2 months later for plastic stent removal. She was in her normal state of health. Both plastic stents were removed uneventfully with a forward-viewing esophagogastroduodenoscope (GIF-H290; Olympus). The pancreatic stent was removed first, followed by the biliary stent, using Rat Tooth Alligator Jaw forceps (MODEL FG-42L-1; Olympus) (Fig. 2).

The patient returned to the hospital $3 \mathrm{~h}$ later, complaining of a fever of $39.4^{\circ} \mathrm{C}$ and abdominal pain. Laboratory testing revealed a white blood cell count of $14,200 / \mathrm{mm}^{3}$ and a pancreatic amylase of $990 \mathrm{U} / \mathrm{L}$. CT with contrast revealed slight inflammation in the pancreatic tail, consistent with mild acute pancreatitis (Fig. 3a). A 10-mm cystic structure consistent with intraductal papillary mucinous neoplasm was observed near the ampulla, but remained largely unchanged from previous studies (Fig. 3b). No biliary or pancreatic duct dilatation was observed.

The abdominal pain resolved completely with intravenous fluids within $2 \mathrm{~h}$. Laboratory data normalized in 4 days. Subsequent magnetic resonance cholangiopancreatography showed several cysts, with no signs of pancreatic duct stenosis (Fig. 4a). Endoscopic ultrasound

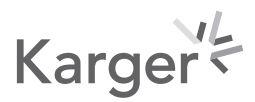




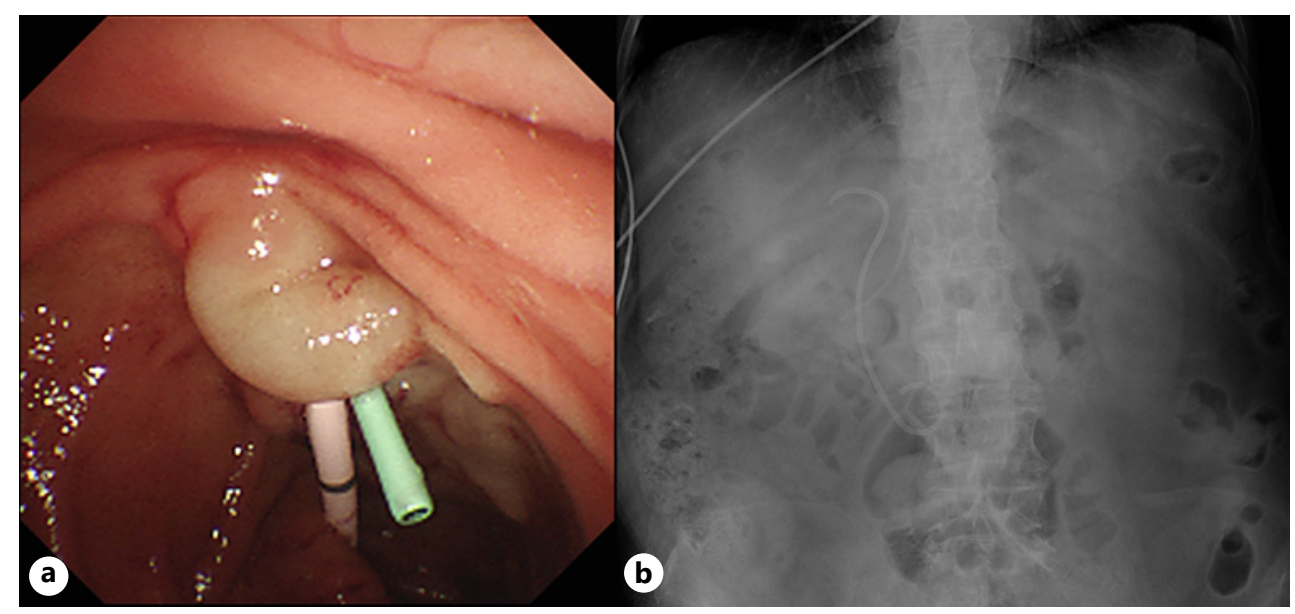

Fig. 1. Biliary and pancreatic stents placed during endoscopic retrograde cholangiopancreatography, confirmed endoscopically (a) and radiographically (b).

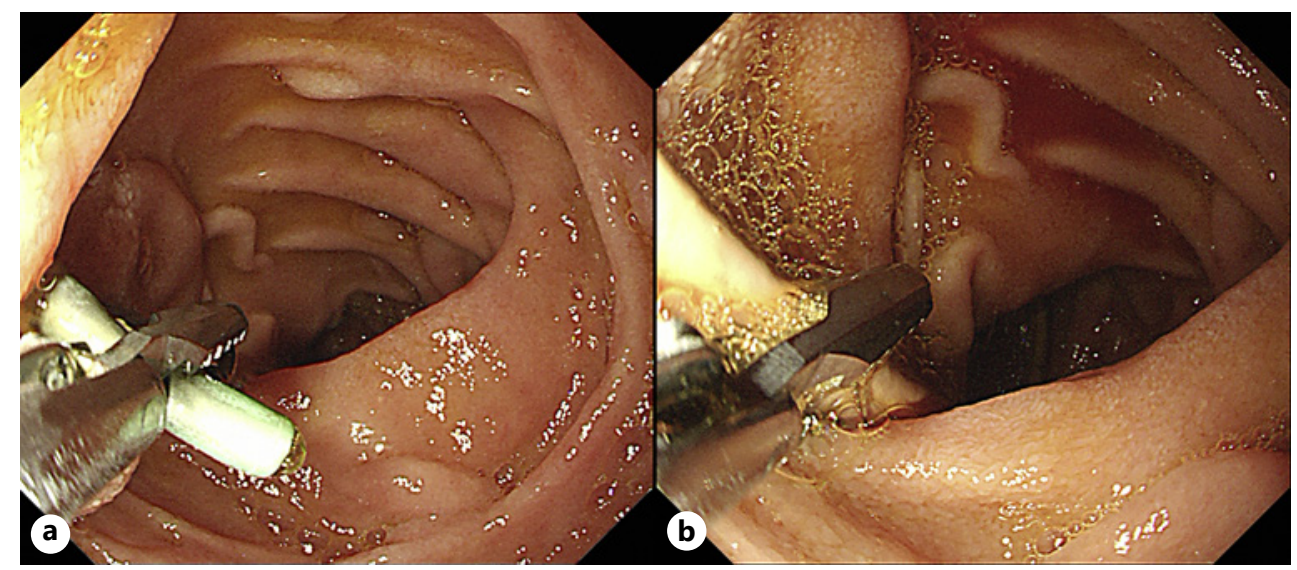

Fig. 2. Endoscopic stent removal. The pancreatic stent was removed first (a), followed by the biliary stent (b).

confirmed a cystic structure in the pancreatic head, with no worrisome features or signs of pancreatic duct obstruction (Fig. 4b). No excretion of mucin from the ampulla was observed. No recurrence was observed during 3 months of follow-up.

\section{Discussion/Conclusion}

Acute pancreatitis occurs after double-balloon enteroscopy in about $0.3 \%$ of cases and rarely after routine colonoscopy [3-5]. However, there are no reports of pancreatitis after simply removing plastic biliary and pancreatic stents which were placed prophylactically in a normal pancreas.

Biliary stents, and less frequently pancreatic stents, are removed and replaced during ERCP with minimal complications. When removed during ERCP, the axis of stents being removed is more or less aligned to the biliary or pancreatic duct. On the other hand, when an esophagogastroduodenoscope is used, stents are pulled out in the direction of the stomach at an acute angle. The stent may therefore apply pressure and cause friction on 
Fig. 3. a Computed tomography with contrast revealed slight inflammation in the pancreatic tail (arrows), consistent with mild acute pancreatitis. b A $10-\mathrm{mm}$ cystic structure (arrow) was observed near the ampulla.

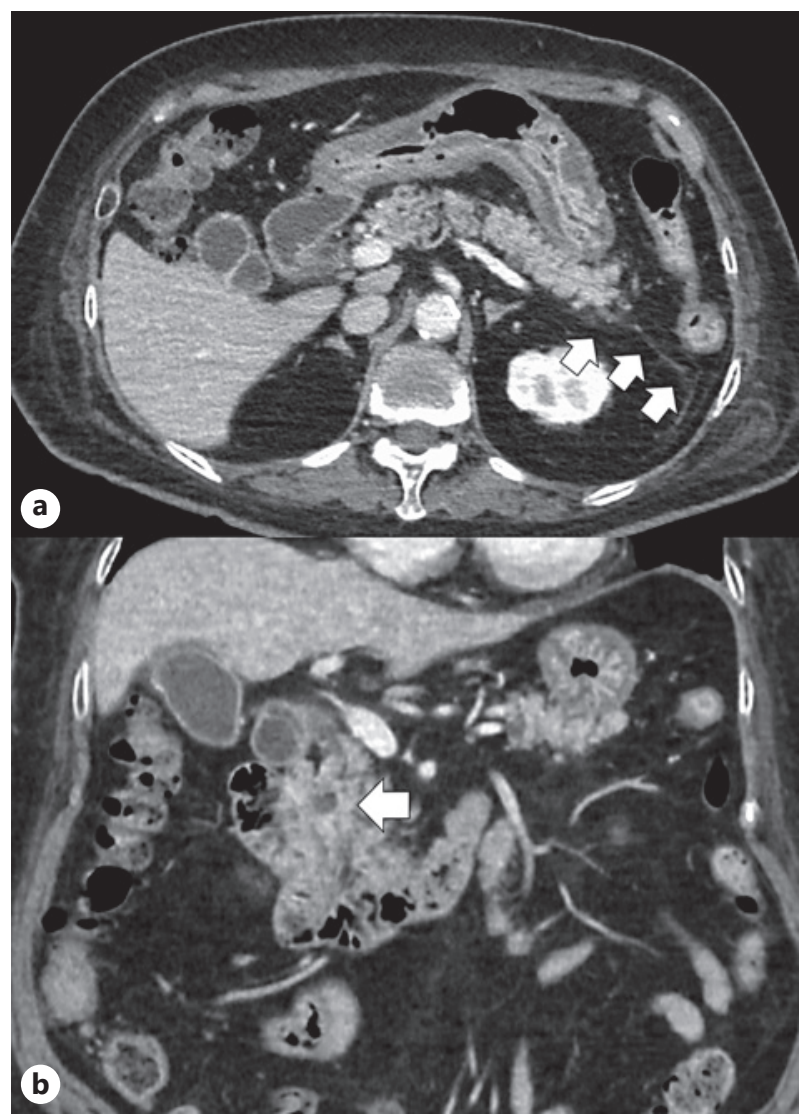

the proximal side of the ampulla during removal. When the distal portion of the stent is removed, the distal end may brush against the distal side of the ampulla. Both may potentially trigger transient papillary edema, leading to temporary pancreatic duct obstruction and pancreatitis.

Given the time lag of 2 months before stent removal in this case, there was concern about stent-induced symptomatic pancreatic duct stricture [6]. This is reported to occur in $2.4 \%$ of patients with normal pancreases after a median stent placement of 7 days and to require total pancreatectomy in severe cases [7]. However, subsequent magnetic resonance cholangiopancreatography showed no stricture in our case, and symptoms resolved in $2 \mathrm{~h}$. We did use a $5-\mathrm{cm}$ pancreatic stent, although a $3-\mathrm{cm}$ stent has been shown to be preferable [8].

Acute pancreatitis is a worrisome feature of IPMN, which has been reported to be a risk factor for high-grade dysplasia in surgical specimens $[9,10]$. A study on history of acute pancreatitis in 182 resected IPMNs revealed that size, location, macroscopic type, and main pancreatic duct diameter were not predictors of acute pancreatitis [11]. Thus, we cannot guarantee that the IPMN played no role in the pathogenesis of acute pancreatitis in this case. However, given that symptoms occurred only $3 \mathrm{~h}$ after stent removal, it appears safe to assume that stent removal was the main cause of pancreatitis in this patient.

In conclusion, we report a case of acute pancreatitis induced by removal of biliary and pancreatic plastic stents. While pancreatic stents are often placed prophylactically during ERCP, this case suggests that there are also risks associated with stent placement. Further studies are required to determine whether a duodenoscope is preferable for biliary and pancreatic stent removal in the ambulatory setting. 
Fig. 4. a Magnetic resonance cholangiopancreatography showed several cysts, with no signs of pancreatic duct stenosis. b Endoscopic ultrasound confirmed a cystic structure in the pancreatic head, with no worrisome features or signs of pancreatic duct obstruction.

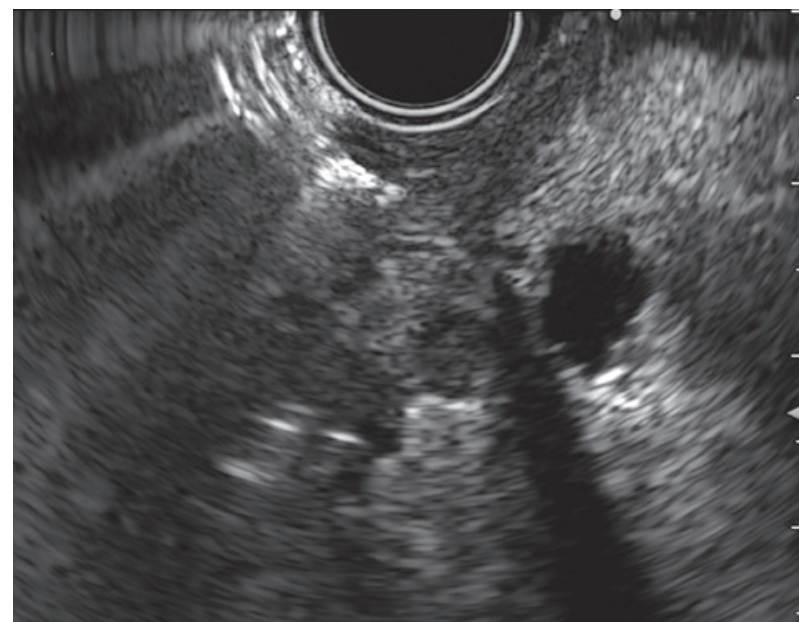

\section{a}

b

\section{Acknowledgment}

This manuscript does not include any nonauthor contributors to acknowledge.

\section{Statement of Ethics}

The patient has given her written informed consent to publish this case (including publication of images). The identity of the patient has been protected. The Ethics Committee at St. Luke's International Hospital does not required ethical approval for case reports.

\section{Conflict of Interest Statement}

The authors have no conflicts of interest to declare.

\section{Funding Sources}

This manuscript did not receive any funding. 
Okamoto and Fukuda: Acute Pancreatitis after Stent Removal

\section{Author Contributions}

Takeshi Okamoto wrote the manuscript. Katsuyuki Fukuda critically reviewed the manuscript. Both authors approved the final version of the manuscript.

\section{Data Availability Statement}

All data generated or analyzed for this case report are included in this article. Further inquiries can be directed to the corresponding author.

\section{References}

1 Sofuni A, Maguchi H, Mukai T, Kawakami H, Irisawa A, Kubota K, et al. Endoscopic pancreatic duct stents reduce the incidence of post-endoscopic retrograde cholangiopancreatography pancreatitis in high-risk patients. Clin Gastroenterol Hepatol. 2011;9:851-8; quiz e110.

2 Fan JH, Qian JB, Wang YM, Shi RH, Zhao CJ. Updated meta-analysis of pancreatic stent placement in preventing post-endoscopic retrograde cholangiopancreatography pancreatitis. World J Gastroenterol. 2015;21:757783.

3 Kopacova M, Tacheci I, Rejchrt S, Bartova J, Bures J. Double balloon enteroscopy and acute pancreatitis. World J Gastroenterol. 2010;16:2331-40.

4 Sidiqi MM, Gong B. Acute pancreatitis as a complication of routine colonoscopy-A rare case report. Int J Surg Case Rep. 2019;57:81-3.

5 Nevins AB, Keeffe EB. Acute pancreatitis after gastrointestinal endoscopy. J Clin Gastroenterol. 2002;34:94-5.

6 Bakman YG, Safdar K, Freeman ML. Significant clinical implications of prophylactic pancreatic stent placement in previously normal pancreatic ducts. Endoscopy. 2009;41:1095-8.

7 Adachi K, Yamauchi H, Kida M, Okuwaki K, Iwai T, Tadehara M, et al. Stent-induced symptomatic pancreatic duct stricture after endoscopic prophylactic pancreatic duct stent placement for the normal pancreas. Pancreatology. 2019;19:665-71.

8 Fujisawa T, Kagawa K, Ochiai K, Hisatomi K, Kubota K, Sato H, et al. Prophylactic efficacy of 3- or 5-cm pancreatic stents for preventing post-ERCP pancreatitis: a prospective, randomized trial. J Clin Gastroenterol. 2016;50: e30-4.

9 Tanaka M, Fernández-Del Castillo C, Kamisawa T, Jang JY, Levy P, Ohtsuka T, et al. Revisions of international consensus Fukuoka guidelines for the management of IPMN of the pancreas. Pancreatology. 2017;17:738-53.

10 Izumo W, Higuchi R, Furukawa T, Yazawa T, Uemura S, Shiihara M, et al. Importance of each high-risk stigmata and worrisome features as a predictor of high-grade dysplasia in intraductal papillary mucinous neoplasms of the pancreas. Pancreatology. 2020;20:895-901.

11 Tanaka T, Masuda A, Sofue K, Toyama H, Shiomi H, Sakai A, et al. Acute pancreatitis in intraductal papillary mucinous neoplasms correlates with pancreatic volume and epithelial subtypes. Pancreatology. 2021;21(1): 138-43.

\section{Karger'א}

